\title{
Research Article \\ Hermitean Cauchy Integral Decomposition of Continuous Functions on Hypersurfaces
}

\author{
Ricardo Abreu Blaya, ${ }^{1}$ Juan Bory Reyes, ${ }^{2}$ Fred Brackx, ${ }^{3}$ Bram De Knock, ${ }^{3}$ \\ Hennie De Schepper, ${ }^{3}$ Dixan Peña Peña, ${ }^{4}$ and Frank Sommen ${ }^{4}$ \\ ${ }^{1}$ Facultad de Informática y Matemática, Universidad de Holguín, Holguín 80100, Cuba \\ ${ }^{2}$ Departamento de Matemática, Universidad de Oriente, Santiago de Cuba 90500, Cuba \\ ${ }^{3}$ Department of Mathematical Analysis, Faculty of Engineering, Ghent University, Galglaan 2, \\ 9000 Ghent, Belgium \\ ${ }^{4}$ Department of Mathematical Analysis, Faculty of Sciences, Ghent University, Galglaan 2, \\ 9000 Ghent, Belgium \\ Correspondence should be addressed to Hennie De Schepper, hds@cage.ugent.be
}

Received 12 August 2008; Accepted 23 October 2008

Recommended by Colin Rogers

We consider Hölder continuous circulant $(2 \times 2)$ matrix functions $G_{2}^{1}$ defined on the AhlforsDavid regular boundary $\Gamma$ of a domain $\Omega$ in $\mathbb{R}^{2 n}$. The main goal is to study under which conditions such a function $G_{2}^{1}$ can be decomposed as $G_{2}^{1}=G_{2}^{1^{+}}-G_{2}^{1^{-}}$, where the components $\mathrm{G}_{2}^{1^{ \pm}}$are extendable to two-sided $\mathrm{H}$-monogenic functions in the interior and the exterior of $\Omega$, respectively. $\mathbf{H}$-monogenicity is a concept from the framework of Hermitean Clifford analysis, a higher dimensional function theory centered around the simultaneous null solutions of two first-order vector-valued differential operators, called Hermitean Dirac operators. H-monogenic functions then are the null solutions of a $(2 \times 2)$ matrix Dirac operator, having these Hermitean Dirac operators as its entries; such functions have been crucial for the development of function theoretic results in the Hermitean Clifford context.

Copyright (C) 2008 Ricardo Abreu Blaya et al. This is an open access article distributed under the Creative Commons Attribution License, which permits unrestricted use, distribution, and reproduction in any medium, provided the original work is properly cited.

\section{Introduction}

Clifford analysis essentially is a higher dimensional function theory offering both a generalization of the theory of holomorphic functions in the complex plane and a refinement of classical multidimensional harmonic analysis. The standard case, nowadays also called Euclidean Clifford analysis, focuses on monogenic functions, that is, the null solutions of the vector-valued Dirac operator $\partial_{\underline{X}}=\sum_{j=1}^{m} e_{j} \partial_{x_{j}}$, factorizing the $m$-dimensional Laplacian: $\partial_{\underline{X}}^{2}=-\Delta_{m}$. Here $\left(e_{1}, \ldots, e_{m}\right)$ is an orthonormal basis for the quadratic space $\mathbb{R}^{0, m}$ underlying the construction of the real Clifford algebra $\mathbb{R}_{0, m}$. The fundamental group leaving the Dirac 
operator invariant is the special orthogonal group $\mathrm{SO}(m ; \mathbb{R})$, doubly covered by the $\operatorname{spin}(m)$ group of the Clifford algebra $\mathbb{R}_{0, m}$. For this reason, the Dirac operator is called a rotation invariant operator. Standard references for Euclidean Clifford analysis are [1-4].

In a series of recent papers, the so-called Hermitean Clifford analysis has emerged as yet a refinement of the Euclidean case. One of the ways for introducing it is by considering the complex Clifford algebra $\mathbb{C}_{2 n}$ and a so-called complex structure on it, that is, an $\mathrm{SO}(2 n ; \mathbb{R})$ element $J$ for which $J^{2}=-\mathbf{1}$. It is precisely the requirement that such a complex structure exists, which forces the dimension of the underlying vector space to be even: $m=2 n$. The resulting function theory focuses on the simultaneous null solutions of two complex Hermitean Dirac operators $\partial_{\underline{Z}}$ and $\partial_{Z^{\dagger}}$ which no longer factorize, but still decompose the Laplace operator in the sense that $4\left(\partial_{\underline{Z}} \partial_{\underline{Z}^{\dagger}}+\partial_{\underline{Z^{\dagger}}} \partial_{\underline{Z}}\right)=\Delta_{2 n}$. The fundamental group symmetry of this system breaks down to the action of the special unitary group. The study of complex Dirac operators was initiated in [5-8]; a systematic development of the associated function theory, including the invariance properties with respect to the underlying Lie groups and Lie algebras, is still in full progress (see, e.g., [9-13]).

In the paper [14], a Cauchy integral formula for Hermitean monogenic functions was established, obviously an essential result in the function theory. However, as in some very particular cases Hermitean monogenicity turns out to be equivalent with anti-holomorphy in $n$ complex variables $\left(z_{1}, \ldots, z_{n}\right)$ (see [11]), it was predictable that such a representation formula could not, in the present setting, take the traditional form as in the complex plane or in Euclidean Clifford analysis. Indeed, a matrix approach had to be followed in order to obtain the desired result, leading to the concept of (left or right) H-monogenic functions, introduced as circulant $(2 \times 2)$ matrix functions, which are (left or right) null solutions of a $(2 \times 2)$ circulant matrix Dirac operator, having the Hermitean Dirac operators $\partial_{\underline{Z}}$ and $\partial_{\underline{Z}^{\dagger}}$ as its entries.

Although the $\mathbf{H}$-monogenic system thus arose as an auxiliary concept in Hermitean Clifford analysis, it deserves to be further studied for its own intrinsic value. In this paper, we consider Hölder continuous circulant $(2 \times 2)$ matrix functions $\mathbf{G}_{2}^{1}$ defined on the AhlforsDavid regular boundary $\Gamma$ of a domain $\Omega$ in $\mathbb{R}^{2 n}$, and we investigate under which conditions such a function $G_{2}^{1}$ can be decomposed as $G_{2}^{1}=G_{2}^{1^{+}}-G_{2}^{1^{-}}$, where the components $G_{2}^{1^{ \pm}}$ are extendable to two-sided $\mathrm{H}$-monogenic functions in the interior and the exterior of $\Omega$, respectively. Such type of decomposition (or "jump") problem was considered already in Euclidean Clifford analysis in, for example, [15].

The present decomposition problem is discussed using the matrix Cauchy integral $\mathcal{C}\left[G_{2}^{1}\right]$ of $\mathbf{G}_{2}^{1}$ (see also [16]) and its singular version $\mathcal{S}\left[\mathrm{G}_{2}^{1}\right]$, called the Hilbert transform; they are shown to be related to each other by Plemelj-Sokhotzki type formulae for the continuous boundary values of $\mathcal{C}\left[G_{2}^{1}\right]$. Moreover, a result is obtained connecting the twosided $\mathbf{H}$-monogenicity of a function $\mathbf{G}_{2}^{1}$ in $\Omega$ to a conservation law for the Hilbert transforms $\mathcal{S}\left[\left.\mathrm{G}_{2}^{1}\right|_{\Gamma}\right]$ and $\left[\left.\mathrm{G}_{2}^{1}\right|_{\Gamma}\right] \mathcal{S}$ of its trace on the boundary.

\section{Preliminaries}

Let $\left(e_{1}, \ldots, e_{m}\right)$ be an orthonormal basis of the Euclidean space $\mathbb{R}^{m}$, and consider the complex Clifford algebra $\mathbb{C}_{m}$ constructed over $\mathbb{R}^{m}$. The noncommutative (also called geometric) multiplication in $\mathbb{C}_{m}$ is governed by the rules

$$
\begin{aligned}
& e_{j}^{2}=-1, \quad j=1, \ldots, m, \\
& e_{j} e_{k}+e_{k} e_{j}=0, \quad j \neq k .
\end{aligned}
$$


The Clifford algebra $\mathbb{C}_{m}$ thus is generated additively by elements of the form $e_{A}=e_{j_{1}} \cdots e_{j_{k}}$, where $A=\left\{j_{1}, \ldots, j_{k}\right\} \subset\{1, \ldots, m\}$ is such that $j_{1}<\cdots<j_{k}$, and so the dimension of $\mathbb{C}_{m}$ is $2^{m}$. For $A=\varnothing$, one puts $e_{\varnothing}=1$, the identity element. Any Clifford number $\lambda \in \mathbb{C}_{m}$ may thus be written as $\lambda=\sum_{A} \lambda_{A} e_{A}, \lambda_{A} \in \mathbb{C}$ and its Hermitean conjugate $\lambda^{\dagger}$ is defined by

$$
\lambda^{\dagger}=\sum_{A} \lambda_{A}^{c} \bar{e}_{A}
$$

where the bar denotes the usual real Clifford algebra conjugation, that is, the main antiinvolution for which $\bar{e}_{j}=-e_{j}$, and ${ }^{c}$ denotes the standard complex conjugation. Note that, as any complex Clifford number $\lambda \in \mathbb{C}_{m}$ may also be written as $\lambda=a+i b, a, b \in \mathbb{R}_{0, m}$, the Hermitean conjugation also takes the form $\lambda^{\dagger}=\bar{a}-i \bar{b}$.

Euclidean space $\mathbb{R}^{m}$ is embedded in the Clifford algebra $\mathbb{C}_{m}$ by identifying $\left(X_{1}, \ldots, X_{m}\right)$ with the real Clifford vector $\underline{X}$ given by

$$
\underline{X}=\sum_{j=1}^{m} e_{j} x_{j}
$$

The square of $\underline{X}$ is scalar-valued and equals the norm squared up to a minus sign: $X^{2}=-|X|^{2}$. The Fischer dual of the vector $\underline{X}$ is the vector-valued first-order differential operator

$$
\partial_{\underline{X}}=\sum_{j=1}^{m} e_{j} \partial_{x_{j}}
$$

called Dirac operator; it is precisely this Dirac operator which underlies the notion of monogenicity of a function, the higher dimensional counterpart of holomorphy in the complex plane. The functions under consideration are defined on an open subset $\Omega$ of $\mathbb{R}^{m}$ and take values in the Clifford algebra $\mathbb{C}_{m}$. They are of the form $g=\sum_{A} g_{A} e_{A}$, where the functions $g_{A}$ are complex-valued. Whenever a property such as continuity, differentiability, and so forth is ascribed to $g$, it is meant that all the components $g_{A}$ possess the cited property. Such function $g$, assumed to be differentiable in $\Omega$, is called left monogenic or right monogenic in $\Omega$ if and only if $\partial_{\underline{X}} g=0$, or $g \partial_{\underline{X}}=0$, respectively. Functions which are both left and right monogenic are called two-sided monogenic. As the Dirac operator factorizes the Laplacian $\Delta_{m}=-\partial_{\underline{X}}^{2}$, monogenicity can be regarded as a refinement of harmonicity. Within the even part of the Clifford algebra, one can realize the spin group, given by

$$
\operatorname{Spin}(m)=\left\{s=\prod_{j=1}^{2 k} \underline{\omega}_{j}: \underline{\omega}_{j} \in S^{m-1}, k \in \mathbb{N}\right\},
$$

where $S^{m-1} \subset \mathbb{R}^{m}$ denotes the unit sphere containing vectors $\underline{\omega}_{j}$ for which $\underline{\omega}_{j}^{2}=-1$. The group Spin $(m)$ yields a double cover for the orthogonal group $\mathrm{SO}(m)$, defined by the map $\chi: \operatorname{Spin}(m) \mapsto \mathrm{SO}(m)$, with $X(s)[\underline{X}]=s \underline{X} \bar{s}$. For a $\mathbb{C}_{m}$-valued function $g(\underline{X})$, the induced action of a spin element $s$ is given by $s g(\bar{s} \underline{X} s) \bar{s}$; it is well known (see [1]) that the Dirac operator commutes with this action, whence we call it rotation invariant. 
The transition from the Euclidean Clifford setting described above to Hermitean Clifford analysis now is essentially based on the introduction of a complex structure $J$. This is a particular element of $\mathrm{SO}(m)$, satisfying $J^{2}=-\mathbf{1}_{m}$. Note that such an element cannot exist when the dimension $m$ of the underlying vector space is odd, whence from now on, we will put $m=2 n$. In terms of our basis, a particular realization of the complex structure is given by $J\left[e_{2 j-1}\right]=-e_{2 j}$ and $J\left[e_{2 j}\right]=e_{2 j-1}, j=1, \ldots, n$. The two projection operators $\pm(1 / 2)\left(\mathbf{1}_{2 n} \pm i J\right)$ associated to this complex structure $J$ then produce the main objects in the Hermitean Clifford setting by acting upon the corresponding objects in the Euclidean one. First of all, the vector space $\mathbb{C}^{2 n}$ thus decomposes as $W^{+} \oplus W^{-}$into two isotropic subspaces.

The real Clifford vector $\underline{X}$ is now denoted as

$$
\underline{X}=\sum_{j=1}^{n}\left(e_{2 j-1} x_{2 j-1}+e_{2 j} x_{2 j}\right)
$$

and the Dirac operator $\partial_{\underline{X}}$ as

$$
\partial_{\underline{X}}=\sum_{j=1}^{n}\left(e_{2 j-1} \partial_{x_{2 j-1}}+e_{2 j} \partial_{x_{2 j}}\right)
$$

while we will also consider their so-called "twisted" counterparts, obtained through the action of $J$, that is,

$$
\begin{gathered}
\underline{X} \mid=\sum_{j=1}^{n}\left(e_{2 j-1} x_{2 j}-e_{2 j} x_{2 j-1}\right), \\
\partial_{\underline{X} \mid}=\sum_{j=1}^{n}\left(e_{2 j-1} \partial_{x_{2 j}}-e_{2 j} \partial_{x_{2 j-1}}\right) .
\end{gathered}
$$

As was the case with $\partial_{X}$, a notion of monogenicity may be associated in a natural way to $\partial_{X \mid}$ as well. Note that the vectors $\underline{X}$ and $\underline{X} \mid$ anticommute, as do the Dirac operators $\partial_{X}$ and $\partial_{\underline{X}} \mid$, while it also holds that $\left.\underline{X}\right|^{2}=\underline{X}^{2}=-|\underline{X}|^{2}$, and $-\partial_{\underline{X} \mid}^{2}=-\partial_{\underline{X}}^{2}=\Delta_{2 n}$. The projections of the vector variable $\underline{X}$ and the Dirac operator $\partial_{\underline{X}}$ on the spaces $W^{ \pm}$then give rise to the Hermitean Clifford variables $\underline{Z}$ and $\underline{Z}^{\dagger}$, given by

$$
\underline{Z}=\frac{1}{2}(\underline{X}+i \underline{X} \mid), \quad \underline{Z}^{\dagger}=-\frac{1}{2}(\underline{X}-i \underline{X} \mid)
$$

and (up to a factor) to the Hermitean Dirac operators $\partial_{\underline{Z}}$ and $\partial_{Z^{\dagger}}$ given by

$$
\partial_{\underline{Z}^{\dagger}}=\frac{1}{4}\left(\partial_{\underline{X}}+i \partial_{\underline{X} \mid}\right), \quad \partial_{\underline{Z}}=-\frac{1}{4}\left(\partial_{\underline{X}}-i \partial_{\underline{X} \mid}\right)
$$


$($ see $[9,10])$. Observe for further use that the Hermitean vector variables and Dirac operators are isotropic, that is, $(\underline{Z})^{2}=\left(\underline{Z}^{\dagger}\right)^{2}=0$ and $\left(\partial_{\underline{Z}}\right)^{2}=\left(\underline{\partial}_{\underline{Z}^{\dagger}}\right)^{2}=0$, whence the Laplacian allows for the decomposition

$$
\Delta_{2 n}=4\left(\partial_{\underline{Z}} \partial_{\underline{Z}^{\dagger}}+\partial_{\underline{Z}^{\dagger}} \partial_{\underline{Z}}\right)
$$

while also

$$
\underline{Z Z} \underline{Z}^{\dagger}+\underline{Z}^{\dagger} \underline{Z}=|\underline{Z}|^{2}=\left|\underline{Z}^{\dagger}\right|^{2}=|\underline{X}|^{2}=\left.|\underline{X}|\right|^{2}
$$

These objects lie at the core of the Hermitean function theory by means of the following definition (see, e.g., $[9,10])$.

Definition 2.1. A continuously differentiable function $g$ in $\Omega \subset \mathbb{R}^{2 n}$ with values in $\mathbb{C}_{2 n}$ is called left Hermitean monogenic (or left h-monogenic for short) in $\Omega$, if and only if it satisfies in $\Omega$ the system

$$
\partial_{\underline{Z}} g=0=\partial_{\underline{Z}^{\dagger}} g
$$

or, equivalently, the system

$$
\partial_{\underline{X}} g=0=\partial_{\underline{X}} g .
$$

In a similar way, right h-monogenicity is defined. Functions which are both left and right h-monogenic are called two-sided h-monogenic.

This definition inspires the statement that h-monogenicity constitutes a refinement of monogenicity.

The main point of difference between the Hermitean framework and the Euclidean one, is the underlying group invariance of the considered Dirac operators. To this end, we consider the group $\operatorname{Spin}_{J}(2 n) \subset \operatorname{Spin}(2 n)$, given by

$$
\operatorname{Spin}_{J}(2 n)=\left\{s \in \operatorname{Spin}(2 n) \mid s s_{J}=s_{J} s\right\},
$$

its definition involving the spin element $s_{J}$ corresponding to the complex structure: $\chi\left(s_{J}\right)=$ $J$. It has been proved that this group constitutes a realization in the Clifford algebra of the unitary group $U(n)$, and moreover, that the Hermitean Dirac operators commute with its associated action. Less precisely, one thus says that these operators are invariant under the action of the unitary group, and so is the notion of h-monogenicity.

\section{A pair of Cauchy integrals and Hilbert transforms in the Euclidean setting}

From now on, we denote by $\Omega$ a Jordan domain in $\mathbb{R}^{2 n}$, and we put $\Omega^{+}=\Omega$ and $\Omega^{-}=\mathbb{R}^{2 n} \backslash \overline{\Omega^{+}}$, where both open sets are assumed to be connected. Furthermore, we assume the boundary $\Gamma$ of $\Omega$ to be a $(2 n-1)$-dimensional compact topological and oriented hypersurface, which 
moreover is Ahlfors-David regular (see [17]). The latter means that there exists a constant $C>0$ such that for all $\underline{X} \in \Gamma$ and all $0<r \leq \operatorname{diam} \Gamma$

$$
C^{-1} r^{2 n-1} \leq \mathscr{\ell}^{2 n-1}(\Gamma \cap \bar{B}(\underline{X} ; r)) \leq C r^{2 n-1},
$$

where $\mathfrak{l}^{2 n-1}$ denotes the $(2 n-1)$-dimensional Hausdorff measure and $\bar{B}(\underline{X} ; r)$ denotes, as usual, the closed ball with radius $r$ and centred at the point $\underline{X}$.

Now take a function $g \in C^{0, \alpha}(\Gamma)$, that is, $g$ is $\alpha$-Hölder continuous on $\Gamma$, with $0<\alpha<1$. We may then consider the Cauchy integrals $C[g]$ and $C \mid[g]$ in $\mathbb{R}^{2 n} \backslash \Gamma$, defined by

$$
\begin{aligned}
& C[g](\underline{X})=\int_{\Gamma} E(\underline{Y}-\underline{X}) \underline{n}(\underline{Y}) g(\underline{Y}) d \mathscr{\ell}^{2 n-1}(\underline{Y}) \\
& C\left|[g](\underline{X})=\int_{\Gamma} E\right|(\underline{Y}-\underline{X}) \underline{n} \mid(\underline{Y}) g(\underline{Y}) d \mathscr{\ell}^{2 n-1}(\underline{Y})
\end{aligned}
$$

as well as their respective singular versions $S[g]$ and $S \mid[g]$ in $\Gamma$, also called Hilbert transforms, defined by

$$
\begin{aligned}
& S[g](\underline{U})=2 \lim _{\varepsilon \rightarrow 0+} \int_{\Gamma \backslash \bar{B}(\underline{U} ; \varepsilon)} E(\underline{Y}-\underline{U}) \underline{n}(\underline{Y})(g(\underline{Y})-g(\underline{U})) d \mathscr{\ell}^{2 n-1}(\underline{Y})+g(\underline{U}), \\
& S\left|[g](\underline{U})=2 \lim _{\varepsilon \rightarrow 0+} \int_{\Gamma \backslash \bar{B}(\underline{U} ; \varepsilon)} E\right|(\underline{Y}-\underline{U}) \underline{n} \mid(\underline{Y})(g(\underline{Y})-g(\underline{U})) d \mathscr{\ell}^{2 n-1}(\underline{Y})+g(\underline{U}) .
\end{aligned}
$$

The so-called Cauchy kernels $E$ and $E \mid$ in the above definitions are derived from the fundamental solutions of the Dirac operators $\partial_{\underline{X}}$ and $\partial_{\underline{X}}$, and are, respectively, given by

$$
E(\underline{X})=-\frac{1}{a_{2 n}} \frac{\underline{X}}{|\underline{X}|^{2 n}}, \quad E \mid(\underline{X})=-\frac{1}{a_{2 n}} \frac{\underline{X} \mid}{|\underline{X}|^{2 n}}
$$

where $a_{2 n}$ denotes the surface area of the unit sphere $S^{2 n-1}$ in $\mathbb{R}^{2 n}$. Furthermore,

$$
\underline{n}(\underline{Y})=\sum_{j=1}^{n}\left(e_{2 j-1} n_{2 j-1}(\underline{Y})+e_{2 j} n_{2 j}(\underline{Y})\right)
$$

stands for the unit normal vector on $\Gamma$ at the point $\underline{Y}$ (as introduced by Federer, see [18]) and $\underline{n} \mid(\underline{Y})$ is its twisted counterpart. Note that $C[g]$ (resp., $C \mid[g]$ ) is left monogenic in $\mathbb{R}^{2 n} \backslash \Gamma$ with regards to the Dirac operator $\partial_{\underline{X}}\left(\right.$ resp., $\left.\partial_{\underline{X} \mid}\right)$ and that

$$
\lim _{|\underline{X}| \rightarrow \infty} C[g](\underline{X})=0=\lim _{|\underline{X}| \rightarrow \infty} C \mid[g](\underline{X}) .
$$


For the sake of completeness, we recall some basic properties of the singular Cauchy integrals $S$ and $S \mid$, which are generalizations to the case of Clifford analysis of the properties established in the complex plane as follows:

(i) $S$ and $S \mid$ are bounded linear operators on $C^{0, \alpha}(\Gamma)(0<\alpha<1)$;

(ii) $S$ and $S \mid$ are involutions on $C^{0, \alpha}(\Gamma)(0<\alpha<1)$, that is, $S^{2}[g]=g$ and $\left.S\right|^{2}[g]=g$ for all $g \in C^{0, \alpha}(\Gamma)$;

(iii) the following Plemelj-Sokhotzki formulae hold for any function $g$ in $C^{0, \alpha}(\Gamma)$ (with $0<\alpha<1)$ :

$$
\begin{aligned}
& C^{ \pm}[g](\underline{U})=\lim _{\Omega^{ \pm} \ni \underline{Y} \rightarrow \underline{U}} C[g](\underline{Y})=\frac{1}{2}(S[g](\underline{U}) \pm g(\underline{U})), \quad \underline{U} \in \Gamma, \\
& \left.C\right|^{ \pm}[g](\underline{U})=\lim _{\Omega^{ \pm} \ni \underline{Y} \rightarrow \underline{U}} C \mid[g](\underline{Y})=\frac{1}{2}(S \mid[g](\underline{U}) \pm g(\underline{U})), \quad \underline{U} \in \Gamma .
\end{aligned}
$$

Formulae (3.7) express the boundary values of the Cauchy integrals in terms of their singular versions. The study of this boundary behaviour, in the Euclidean Clifford analysis context, has been the subject of intensive research in the last years, see for example [19-23]. We must remark that these formulae also hold for a wider class of rectifiable surfaces, containing as proper subclasses for instance differentiable, chord-arc, piecewise smooth, Liapunov, and Lipschitz surfaces as well as simple Lipschitz graphs.

From the above properties, it is clear that the singular Cauchy integral $S$ gives rise to two important operators, that is,

$$
P=\frac{1}{2}(\mathbf{1}+S), \quad Q=\frac{1}{2}(\mathbf{1}-S),
$$

which are mutually complementary projection operators on the same space: $P^{2}=P, Q^{2}=Q$, and $P Q=Q P=0$. The same holds for $S \mid$, where we introduce

$$
P\left|=\frac{1}{2}(\mathbf{1}+S \mid), \quad Q\right|=\frac{1}{2}(\mathbf{1}-S \mid) .
$$

\section{The Hermitean Cauchy integral: a matrix approach}

Starting from the pair of fundamental solutions $(E, E \mid)$ of the Euclidean Dirac operators $\partial_{\underline{X}}$ and $\partial_{\underline{X}}$, we now construct the distributions $\mathcal{\varepsilon}=-(E+i E \mid)$ and $\mathcal{E}^{\dagger}=(E-i E \mid)$. Explicitly they are given by

$$
\varepsilon\left(\underline{Z}, \underline{Z}^{\dagger}\right)=\frac{2}{a_{2 n}} \frac{\underline{Z}}{|\underline{Z}|^{2 n}}, \quad \mathcal{E}^{\dagger}\left(\underline{Z}, \underline{Z}^{\dagger}\right)=\frac{2}{a_{2 n}} \frac{\underline{Z}^{\dagger}}{|\underline{Z}|^{2 n}}
$$

with

$$
\lim _{|\underline{Z}| \rightarrow \infty} \mathcal{E}\left(\underline{Z}, \underline{Z}^{\dagger}\right)=0=\lim _{|\underline{Z}| \rightarrow \infty} \mathcal{E}^{\dagger}\left(\underline{Z}, \underline{Z}^{\dagger}\right)
$$


Note that $\mathcal{\varepsilon}$ and $\mathcal{E}^{\dagger}$ are not the fundamental solutions to the respective Hermitean Dirac operators $\partial_{\underline{Z}}$ and $\partial_{\underline{Z}^{\dagger}}$, but surprisingly, introducing the particular circulant $(2 \times 2)$ matrices

$$
\boldsymbol{\Phi}_{\left(\underline{Z}, \underline{Z}^{\dagger}\right)}=\left(\begin{array}{cc}
\partial_{\underline{Z}} & \partial_{\underline{Z}^{\dagger}} \\
\partial_{\underline{Z}^{\dagger}} & \partial_{\underline{Z}}
\end{array}\right), \quad \boldsymbol{\varepsilon}=\left(\begin{array}{cc}
\varepsilon & \varepsilon^{\dagger} \\
\mathcal{\varepsilon}^{\dagger} & \varepsilon
\end{array}\right), \quad \delta=\left(\begin{array}{ll}
\delta & 0 \\
0 & \delta
\end{array}\right),
$$

where $\delta$ is the Dirac delta distribution, one obtains that $\boldsymbol{\vartheta}_{\left(\underline{Z}, \underline{Z}^{\dagger}\right)} \boldsymbol{\varepsilon}(\underline{Z})=\boldsymbol{\delta}(\underline{Z})$, so that $\boldsymbol{\varepsilon}$ may be considered as a fundamental solution of the operator $\boldsymbol{\Phi}_{\left(\underline{Z}, \underline{Z}^{\dagger}\right)}$ in a matricial context. It was exactly this simple observation which has lead to the idea of following a matrix approach in order to establish a Cauchy integral formula and the related function theoretic results in the Hermitean Clifford setting, see [14, 16]. Moreover, it inspired the following definition.

Definition 4.1. Let $g_{1}, g_{2}$ be continuously differentiable functions defined in $\Omega$ and taking values in $\mathbb{C}_{2 n}$, and consider the matrix function

$$
\mathbf{G}_{2}^{1}=\left(\begin{array}{ll}
g_{1} & g_{2} \\
g_{2} & g_{1}
\end{array}\right)
$$

Then $\mathbf{G}_{2}^{1}$ is called left (resp., right) $\mathbf{H}$-monogenic in $\Omega$ if and only if it satisfies in $\Omega$ the system

$$
\boldsymbol{\Phi}_{\left(\underline{Z}, \underline{Z}^{\dagger}\right)} \mathbf{G}_{2}^{1}=\mathbf{O} \quad\left(\text { resp., } \mathbf{G}_{2}^{1} \boldsymbol{\Phi}_{\left(\underline{Z}, \underline{Z}^{\dagger}\right)}=\mathbf{O}\right)
$$

Here $\mathbf{O}$ denotes the matrix with zero entries.

Explicitly, the system for left $\mathbf{H}$-monogenicity reads

$$
\begin{aligned}
& \partial_{\underline{Z}}\left[g_{1}\right]+\partial_{\underline{Z}^{\dagger}}\left[g_{2}\right]=0, \\
& \partial_{\underline{Z}^{\dagger}}\left[g_{1}\right]+\partial_{\underline{Z}}\left[g_{2}\right]=0 .
\end{aligned}
$$

Note that we have found above that $\boldsymbol{\varepsilon}$ is left (and in fact also right) $\mathbf{H}$-monogenic in $\mathbb{R}^{2 n} \backslash\{\underline{0}\}$.

In general, the $\mathbf{H}$-monogenicity of the matrix function $\mathbf{G}_{2}^{1}$ does not imply the $\mathrm{h}$ monogenicity of its entry functions $g_{1}$ and $g_{2}$. However, choosing in particular $g_{1}=g$ and $g_{2}=0$, the $\mathbf{H}$-monogenicity of the corresponding diagonal matrix $\mathbf{G}_{0}$ is seen to be equivalent to the h-monogenicity of the function $g$.

Defining the matrix Laplacian by

$$
\Delta=\left(\begin{array}{cc}
\Delta_{2 n} & 0 \\
0 & \Delta_{2 n}
\end{array}\right)
$$

we may call the matrix function $\mathrm{G}_{2}^{1}$ harmonic in the domain $\Omega$ if and only if it satisfies the equation $\Delta \mathbf{G}_{2}^{1}=\mathbf{O}$. It is a simple, yet remarkable, fact that the Dirac matrix $\boldsymbol{\Phi}_{\left(\underline{z}, \underline{Z}^{\dagger}\right)}$ still in 
some sense "factorizes the Laplacian" (as does the Cauchy-Riemann operator in the complex plane) since

$$
4 \boldsymbol{\boldsymbol { \vartheta }}_{\left(\underline{Z}, \underline{Z}^{\dagger}\right)}\left(\boldsymbol{\Phi}_{\left(\underline{Z}, \underline{Z}^{\dagger}\right)}\right)^{\dagger}=4\left(\boldsymbol{\Phi}_{\left(\underline{Z}, \underline{Z}^{\dagger}\right)}\right)^{\dagger} \boldsymbol{\Phi}_{\left(\underline{Z}, \underline{Z}^{\dagger}\right)}=\left(\begin{array}{cc}
\Delta_{2 n} & 0 \\
0 & \Delta_{2 n}
\end{array}\right) .
$$

This property guarantees that any $\mathbf{H}$-monogenic matrix function $\mathbf{G}_{2}^{1}$ also is harmonic in $\Omega$, and moreover, its entries are harmonic in the classical sense.

The above matrix approach will form the key towards the construction of a boundary value theory of h-monogenic functions. In what follows, we will restrict to left monogenicity, unless explicitly stated.

From now on the notations $\underline{Y}$ and $\underline{Y}$ | are reserved for Clifford vectors associated to points in $\Omega^{ \pm}$. Their Hermitean counterparts are denoted by

$$
\underline{V}=\frac{1}{2}(\underline{Y}+i \underline{Y} \mid), \quad \underline{V}^{\dagger}=-\frac{1}{2}(\underline{Y}-i \underline{Y} \mid)
$$

while the Hermitean vector pair $\left(\underline{Z}, \underline{Z}^{\dagger}\right)$ still corresponds, as before, to the orthogonal pair $(\underline{X}, \underline{X} \mid)$.

Given functions $g_{1}, g_{2} \in C^{0, \alpha}(\Gamma)(0<\alpha<1)$, we then introduce the vector space

$$
\mathcal{C}^{0, \alpha}(\Gamma)=\left\{\mathbf{G}_{2}^{1}=\left(\begin{array}{ll}
g_{1} & g_{2} \\
g_{2} & g_{1}
\end{array}\right): g_{1}, g_{2} \in C^{0, \alpha}(\Gamma)\right\}
$$

and we define, for $\mathbf{G}_{2}^{1} \in \mathcal{C}^{0, \alpha}(\Gamma)$, its Hermitean matrix Cauchy integral $\mathcal{C}\left[\mathrm{G}_{2}^{1}\right]$ to be

$$
\mathcal{C}\left[\mathrm{G}_{2}^{1}\right](\underline{Y})=\int_{\Gamma} \mathcal{E}\left(\underline{Z}-\underline{V}, \underline{Z}^{\dagger}-\underline{V}^{\dagger}\right) \mathbf{N}_{\left(\underline{Z}, \underline{Z}^{\dagger}\right)} \mathrm{G}_{2}^{1}(\underline{X}) \mathrm{d} \mathscr{\ell}^{2 n-1}, \quad \underline{Y} \in \Omega^{ \pm}
$$

which is H-monogenic in $\Omega^{ \pm}$, that is, $\boldsymbol{\Phi}_{\left(\underline{V}, \underline{V}^{\dagger}\right)} \mathcal{C}\left[\mathbf{G}_{2}^{1}\right](\underline{Y})=\mathbf{O}$ in $\Omega^{ \pm}$. Here we have introduced the additional circulant matrix

$$
\mathbf{N}_{\left(\underline{Z}, \underline{Z}^{\dagger}\right)}=\left(\begin{array}{cc}
\underline{N} & -\underline{N^{\dagger}} \\
-\underline{N} & \underline{N}
\end{array}\right)
$$

containing (up to a factor) the Hermitean projections $\underline{N}$ and $\underline{N}^{\dagger}$ of the outward unit normal vector $\underline{n}(\underline{X})$ at the point $\underline{X}$, given by

$$
\begin{aligned}
\underline{N} & =-\frac{1}{4}(-1)^{n(n+1) / 2}(2 i)^{n}(\underline{n}(\underline{X})-i \underline{n} \mid(\underline{X})), \\
\underline{N}^{\dagger} & =-\frac{1}{4}(-1)^{n(n+1) / 2}(2 i)^{n}(\underline{n}(\underline{X})+i \underline{n} \mid(\underline{X})),
\end{aligned}
$$


and the matrix Hausdorff measure

$$
\mathrm{d} \mathscr{\ell}^{2 n-1}=\left(\begin{array}{cc}
d \mathscr{L}^{2 n-1} & 0 \\
0 & d \mathscr{\ell}^{2 n-1}
\end{array}\right)
$$

A direct calculation reveals that the Hermitean Cauchy integral can be expressed in terms of the Euclidean Cauchy integrals $C$ and $C \mid$ as follows:

$$
\mathcal{C}\left[\mathbf{G}_{2}^{1}\right]=\frac{(-1)^{n(n+1) / 2}(2 i)^{n}}{2}\left(\begin{array}{cc}
C\left[g_{1}-g_{2}\right]+C \mid\left[g_{1}+g_{2}\right] & -C\left[g_{1}-g_{2}\right]+C \mid\left[g_{1}+g_{2}\right] \\
-C\left[g_{1}-g_{2}\right]+C \mid\left[g_{1}+g_{2}\right] & C\left[g_{1}-g_{2}\right]+C \mid\left[g_{1}+g_{2}\right]
\end{array}\right) .
$$

In particular, for the special case of the matrix function $\mathrm{G}_{0}$ (i.e., $g_{1}=g$ and $g_{2}=0$ ) this is reduced to

$$
\mathcal{C}\left[\mathbf{G}_{0}\right]=\frac{(-1)^{n(n+1) / 2}(2 i)^{n}}{2}\left(\begin{array}{cc}
C[g]+C \mid[g] & -C[g]+C \mid[g] \\
-C[g]+C \mid[g] & C[g]+C \mid[g]
\end{array}\right) .
$$

Remark 4.2. It is clear that, in general, $\mathcal{C}\left[\mathbf{G}_{0}\right]$ will not turn out to be a diagonal matrix, whence its entries will not be h-monogenic functions. The particular situation where $C[g]=C \mid[g]$, giving rise to an interpretation in terms of h-monogenicity, is explicitly treated below.

We aim at establishing a generalization of the Plemelj-Sokhotzki formulae to the case of $\mathbf{H}$-monogenic matrix functions. To that end, and at the same time inspired by the structure of the above expressions (4.15)-(4.16), let us introduce the singular matrix Cauchy integral

$$
\mathcal{S}=\frac{1}{2}\left(\begin{array}{cc}
S+S \mid & -S+S \mid \\
-S+S \mid & S+S \mid
\end{array}\right)
$$

its action on the matrix functions $\mathrm{G}_{2}^{1}$ and $\mathrm{G}_{0}$ being given by matrix multiplication, followed by an operator action on the level of the entries, that is,

$$
\begin{aligned}
\mathcal{S}\left[\mathbf{G}_{2}^{1}\right] & =\frac{1}{2}\left(\begin{array}{cc}
S+S \mid & -S+S \mid \\
-S+S \mid & S+S \mid
\end{array}\right)\left(\begin{array}{ll}
g_{1} & g_{2} \\
g_{2} & g_{1}
\end{array}\right) \\
& =\frac{1}{2}\left(\begin{array}{cc}
S\left[g_{1}-g_{2}\right]+S \mid\left[g_{1}+g_{2}\right] & -S\left[g_{1}-g_{2}\right]+S \mid\left[g_{1}+g_{2}\right] \\
-S\left[g_{1}-g_{2}\right]+S \mid\left[g_{1}+g_{2}\right] & S\left[g_{1}-g_{2}\right]+S \mid\left[g_{1}+g_{2}\right]
\end{array}\right) .
\end{aligned}
$$

Invoking the expressions (4.15)-(4.16) for $\mathcal{C}\left[\mathrm{G}_{2}^{1}\right]$ in terms of $C\left[g_{1}-g_{2}\right]$ and $C \mid\left[g_{1}+g_{2}\right]$, and taking into account the Plemelj-Sokhotzki formulae (3.7), the following result is then readily obtained. 
Theorem 4.3. Let $\mathbf{G}_{2}^{1} \in \mathcal{C}^{0, \alpha}(\Gamma)(0<\alpha<1)$, then the continuous boundary values of its Hermitean Cauchy integral $\mathcal{C}\left[\mathbf{G}_{2}^{1}\right]$ exist and are given by

$$
\mathcal{C}^{ \pm}\left[\mathbf{G}_{2}^{1}\right](\underline{U})=\lim _{\substack{\underline{Y} \in \Omega^{ \pm} \\ \underline{U}}} \mathcal{C}\left[\mathbf{G}_{2}^{1}\right](\underline{Y})=(-1)^{n(n+1) / 2}(2 i)^{n}\left( \pm \frac{1}{2} \mathbf{G}_{2}^{1}(\underline{U})+\frac{1}{2} \mathcal{S}\left[\mathbf{G}_{2}^{1}\right](\underline{U})\right), \quad \underline{U} \in \Gamma .
$$

Moreover, some properly adapted analogues of the basic properties of $S$ and $S \mid$, mentioned in the previous section, hold for the matrix operator $\mathcal{S}$.

Theorem 4.4. The singular Hermitean Cauchy integral $\mathcal{S}$ satisfies the following properties:

(i) $\mathcal{S}$ is a bounded linear operator on $\mathcal{C}^{0, \alpha}(\Gamma)(0<\alpha<1)$;

(ii) $\mathcal{S}$ is an involution on $\mathcal{C}^{0, \alpha}(\Gamma)(0<\alpha<1)$, that is, $\mathcal{S}^{2}=\supset$, where $\supset$ is the $(2 \times 2)$ identity matrix operator.

Similarly we put

$$
\boldsymbol{p}=\frac{1}{2}\left(\begin{array}{cc}
P+P \mid & -P+P \mid \\
-P+P \mid & P+P \mid
\end{array}\right), \quad \mathcal{Q}=\frac{1}{2}\left(\begin{array}{cc}
Q+Q \mid & -Q+Q \mid \\
-Q+Q \mid & Q+Q \mid
\end{array}\right),
$$

where the operators $P$ and $Q$ were introduced in (3.8) and the operators $P \mid$ and $Q \mid$ in (3.9). It is directly seen that $\boldsymbol{D}+\boldsymbol{Q}=\mathcal{\supset}$, with $\boldsymbol{D}=(1 / 2)(\supset+\mathcal{S})$ and $\mathcal{Q}=(1 / 2)(\supset-\mathcal{S})$. The following result is then obtained.

Theorem 4.5. The operators $\boldsymbol{D}$ and $\mathcal{Q}$ are mutually complementary projection operators on the same space, that is, $\boldsymbol{D}^{2}=\boldsymbol{D}, \boldsymbol{Q}^{2}=\mathcal{Q}$ and $\boldsymbol{D Q}=\boldsymbol{Q} \boldsymbol{D}=\mathbf{O}$.

This theorem entails the direct decomposition

$$
\mathcal{C}^{0, \alpha}(\Gamma)=\boldsymbol{D}\left[\mathcal{C}^{0, \alpha}(\Gamma)\right] \oplus \mathcal{Q}\left[\mathcal{C}^{0, \alpha}(\Gamma)\right], \quad(0<\alpha<1),
$$

so that each function $\mathrm{G}_{2}^{1} \in \mathcal{C}^{0, \alpha}(\Gamma)$ admits a unique decomposition into components belonging to $\boldsymbol{D}\left[\mathcal{C}^{0, \alpha}(\Gamma)\right]$ and $\mathcal{Q}\left[\mathcal{C}^{0, \alpha}(\Gamma)\right]$, respectively. In what follows, we will use the notations

$$
\mathcal{C}_{+, l}^{0, \alpha}(\Gamma) \equiv \boldsymbol{D}\left[\mathcal{C}^{0, \alpha}(\Gamma)\right], \quad \mathcal{C}_{-, l}^{0, \alpha}(\Gamma) \equiv \mathcal{Q}\left[\mathcal{C}^{0, \alpha}(\Gamma)\right],
$$

when dealing with left $\mathbf{H}$-monogenic functions. Likewise, in the case of right $\mathbf{H}$-monogenicity, we will use $\mathcal{C}_{+, r}^{0, \alpha}(\Gamma)$ and $\mathcal{C}_{-, r}^{0, \alpha}(\Gamma)$.

\section{The jump problem for $\mathrm{H}$-monogenic functions}

In this section, we will study the so-called jump (or decomposition) problem for left $\mathbf{H}$ monogenic functions, that is, we will investigate under which conditions a given matrix function $\mathrm{G}_{2}^{1}$ can be decomposed as

$$
\mathbf{G}_{2}^{1}=\mathbf{G}_{2}^{1^{+}}-\mathbf{G}_{2}^{1^{-}}
$$


where the components $\mathrm{G}_{2}^{1^{ \pm}}$are extendable to left (resp., right) H-monogenic functions in $\Omega^{ \pm}$, vanishing at infinity. First, it should be noted that if this jump problem has a solution, then it is necessarily unique. This assertion can easily be proved using the Painlevé and Liouville theorems in the Clifford analysis setting, see $[1,15]$. Next, under the condition that $\mathbf{G}_{2}^{1} \in$ $\mathcal{C}^{0, \alpha}(\Gamma)(0<\alpha<1)$, Theorem 4.3 ensures the solvability of the jump problem (5.1) for left $\mathrm{H}$-monogenic functions, its unique solution is given by

$$
\mathbf{G}_{2}^{ \pm}=\frac{1}{(-1)^{n(n+1) / 2}(2 i)^{n}} \mathcal{C}^{ \pm}\left[\mathbf{G}_{2}^{1}\right]
$$

The solvability for right $\mathbf{H}$-monogenic functions can be formulated similarly.

Now consider the special case of the matrix function $\mathbf{G}_{0}$, or equivalently, of a single nonzero entry $g$. The above decomposition problem (5.1) then obviously is strongly connected to the analogous problem for h-monogenic functions, as studied in [24]. In order to be able to rephrase the obtained result (5.2) in the h-monogenic setting, we only need to ensure that $\mathcal{C}\left[\mathbf{G}_{0}\right]$ is interpretable as an h-monogenic function. In view of this observation and of [24, Remark 1, Theorem 2.2] may be reformulated into the present setting as follows.

Theorem 5.1. Let $g \in C^{0, \alpha}(\Gamma)(0<\alpha<1)$ and consider the corresponding matrix function $\mathbf{G}_{0} \in$ $\mathcal{C}^{0, \alpha}(\Gamma)$. Then the jump problem (5.1) is solvable in terms of h-monogenic functions if and only if

$$
\mathcal{C}\left[\mathbf{G}_{0}\right]=\frac{(-1)^{n(n+1) / 2}(2 i)^{n}}{2}\left(\begin{array}{cc}
C[g]+C \mid[g] & 0 \\
0 & C[g]+C \mid[g]
\end{array}\right) .
$$

Proof. Clearly (5.3) is equivalent to the requirement that $C[g]=C \mid[g]$, and thus $S[g]=S \mid[g]$. The remaining entry $C[g]+C|[g]=2 C[g]=2 C|[g]$ of $\mathcal{C}\left[\mathbf{G}_{0}\right]$ then automatically is an hmonogenic function in $\Omega^{ \pm}$.

The next result deals with a necessary and sufficient condition for the extendability of a given matrix function on the hypersurface $\Gamma$ to an $\mathbf{H}$-monogenic function in $\Omega^{+}$or in $\Omega^{-}$, vanishing at infinity. It is clear that the answer will have to involve the projection operators $\boldsymbol{D}$ and $\boldsymbol{Q}$.

Theorem 5.2. Let $\mathbf{G}_{2}^{1} \in \mathcal{C}^{0, \alpha}(\Gamma)(0<\alpha<1)$.

(i) In order for $\mathbf{G}_{2}^{1}$ to be the boundary value of a matrix function $\mathbf{G}_{2}^{1^{+}}$which is $\mathbf{H}$-monogenic in $\Omega^{+}$, it is necessary and sufficient that

$$
\mathbf{G}_{2}^{1} \in i m p
$$

that is, there exists a matrix function $\mathbf{F}_{2}^{1} \in \mathcal{C}^{0, \alpha}(\Gamma)$ such that $\mathbf{G}_{2}^{1}=\boldsymbol{D}\left[\mathbf{F}_{2}^{1}\right]$.

(ii) In order for $\mathbf{G}_{2}^{1}$ to be the boundary value of a matrix function $\mathbf{G}_{2}^{1^{-}}$which is $\mathbf{H}$-monogenic in $\Omega^{-}$and vanishes at infinity, it is necessary and sufficient that

$$
\mathbf{G}_{2}^{1} \in i m \boldsymbol{Q},
$$

that is, there exists a matrix function $\mathbf{F}_{2}^{1} \in \mathcal{C}^{0, \alpha}(\Gamma)$ such that $\mathbf{G}_{2}^{1}=\boldsymbol{Q}\left[\mathbf{F}_{2}^{1}\right]$. 
Proof. First, let $\mathrm{G}_{2}^{1} \in \mathcal{C}^{0, \alpha}(\Gamma)$ be the boundary value of an $\mathrm{H}$-monogenic function $\mathrm{G}_{2}^{1^{+}}$in $\Omega^{+}$. Then, as was already shown in [14], $\mathbf{G}_{2}^{+}$is nothing but the Cauchy integral of $\mathbf{G}_{2}^{1}$, namely,

$$
\mathrm{G}_{2}^{1^{+}}(\underline{Y})=\mathcal{C}\left[\mathrm{G}_{2}^{1}\right](\underline{Y})
$$

Now let $\underline{U} \in \Gamma$ and let $\Omega^{ \pm} \ni \underline{Y} \rightarrow \underline{U}$. Then $\mathrm{G}_{2}^{1^{+}}(\underline{Y}) \rightarrow \mathrm{G}_{2}^{1}(\underline{U})$, while, according to Theorem $\overline{4.3}, \mathcal{C}\left[\mathrm{G}_{2}^{1}\right](\underline{Y}) \rightarrow \mathcal{D}\left[\mathrm{G}_{2}^{1}\right](\underline{\underline{U}})$. Thus, $\mathrm{G}_{2}^{1}(\underline{U})=\underline{\mathcal{D}}\left[\mathrm{G}_{2}^{1}\right](\underline{U})$, yielding (5.4). Conversely, assume that (5.4) holds and consider $\mathrm{G}_{2}^{1^{+}}(\underline{Y})$ given by (5.2), for $\underline{Y} \in \Omega^{+}$. Then $\mathrm{G}_{2}^{1^{+}}$is an $\mathbf{H}-$ monogenic function in $\Omega^{+}$and again by Theorem 4.3 , we have that $\left.\mathrm{G}_{2}^{1^{+}}\right|_{\Gamma}=P\left[\mathrm{G}_{2}^{1}\right]=\mathrm{G}_{2}^{1}$, which proves (i). Similar considerations apply to (ii).

Remark 5.3. Condition (5.4) can be rewritten as

$$
\mathrm{G}_{2}^{1}(\underline{U})=\mathcal{S}\left[\mathrm{G}_{2}^{1}\right](\underline{U}), \quad \underline{U} \in \Gamma,
$$

and condition (5.5) as

$$
\mathrm{G}_{2}^{1}(\underline{U})=-\mathcal{S}\left[\mathrm{G}_{2}^{1}\right](\underline{U}), \quad \underline{U} \in \Gamma .
$$

Remark 5.4. For the special case of the matrix function $\mathrm{G}_{0} \in \mathcal{C}^{0, \alpha}(\Gamma)(0<\alpha<1)$, condition (5.7) can be rephrased in terms of the entry function $g$ as $S[g]=S \mid[g]=g$, which exactly is the criterion for the existence of an h-monogenic extension of $g$ to $\Omega^{+}$, obtained in [24]. Similarly, in $\Omega^{-},(5.8)$ yields $S[g]=S \mid[g]=-g$.

As an application of Theorem 5.2, we consider the Dirichlet boundary value problem for the operator $\boldsymbol{\Phi}_{\left(\underline{Z}, \underline{Z}^{\dagger}\right)}$, which is stated as follows.

Dirichlet problem

Given $\mathbf{G}_{2}^{1} \in \mathcal{C}^{0, \alpha}(\Gamma)(0<\alpha<1)$, find a function $\mathbf{F}_{2}^{1}$ such that

$$
\begin{gathered}
\boldsymbol{\Phi}_{\left(\underline{V}, \underline{V}^{\dagger}\right)}\left[\mathbf{F}_{2}^{1}\right](\underline{Y})=\mathbf{O}, \quad \text { in } \Omega, \\
\mathbf{F}_{2}^{1}=\mathbf{G}_{2^{\prime}}^{1} \quad \text { on } \Gamma .
\end{gathered}
$$

From Theorem 5.2, we immediately see that a solution to this Dirichlet problem will not always exist, as not all functions $\mathbf{G}_{2}^{1}$ are extendable to an $\mathbf{H}$-monogenic function in $\Omega$. Indeed, to this end, they need to satisfy condition (5.4) or equivalently, condition (5.7). If this is fulfilled, the solution of the Dirichlet problem will be given, up to a multiplicative constant, by the Cauchy integral of $\mathbf{G}_{2}^{1}$, namely,

$$
\mathbf{F}_{2}^{1}=\frac{1}{(-1)^{n(n+1) / 2}(2 i)^{n}} \mathcal{C}\left[\mathbf{G}_{2}^{1}\right]
$$




\section{A conservation law for two-sided H-monogenic functions}

This section is devoted to the proof of a remarkable result, establishing a connection between two-sided $\mathbf{H}$-monogenicity of a function $\mathrm{G}_{2}^{1}$ in a domain $\Omega$ and the singular Cauchy integrals $\mathcal{S}\left[\left.\mathrm{G}_{2}^{1}\right|_{\Gamma}\right]$ and $\left[\left.\mathrm{G}_{2}^{1}\right|_{\Gamma}\right] \mathcal{S}$ of its trace on the boundary $\Gamma$ of $\Omega$. We still mention that, in the context of Euclidean Clifford analysis, a similar "conservation law" was obtained in [25] for twosided monogenicity.

Theorem 6.1. Let $\mathbf{G}_{2}^{1} \in \mathcal{C}^{0, \alpha}(\Omega \cup \Gamma)(0<\alpha<1)$, such that $\boldsymbol{\Phi}_{\left(\underline{V}, \underline{V}^{\dagger}\right)}\left[\mathbf{G}_{2}^{1}\right](\underline{Y})=\mathbf{O}$ in $\Omega$. Then the following statements are equivalent:

(i) $\mathbf{G}_{2}^{1}$ is two-sided $\mathbf{H}$-monogenic in $\Omega$;

(ii) $\mathcal{S}\left[\left.\mathrm{G}_{2}^{1}\right|_{\Gamma}\right]=\left[\left.\mathrm{G}_{2}^{1}\right|_{\Gamma}\right] \mathcal{S}$.

Proof. Suppose that, next to its already assumed left $\mathbf{H}$-monogenicity, $\mathbf{G}_{2}^{1}$ also is right $\mathbf{H}$ monogenic in $\Omega$. Then it holds that $\left.\mathrm{G}_{2}^{1}\right|_{\Gamma} \in \mathcal{C}_{+, l, r}^{0, \alpha}(\Gamma)$, whence

$$
\mathcal{S}\left[\left.\mathrm{G}_{2}^{1}\right|_{\Gamma}\right]=\left.\mathrm{G}_{2}^{1}\right|_{\Gamma}=\left[\left.\mathrm{G}_{2}^{1}\right|_{\Gamma}\right] \mathcal{S}
$$

Conversely, suppose that $\mathcal{S}\left[\left.\mathrm{G}_{2}^{1}\right|_{\Gamma}\right]=\left[\left.\mathrm{G}_{2}^{1}\right|_{\Gamma}\right] \mathcal{S}$. From the assumed left $\mathbf{H}$-monogenicity of $\mathbf{G}_{2}^{1}$, we have that $\mathbf{G}_{2}^{1}=\mathcal{C}\left[\left.G_{2}^{1}\right|_{\Gamma}\right]$ and that for $\underline{U} \in \Gamma$ the boundary value taken from the inside, denoted by $\mathrm{BV}^{+}$, is given by

$$
\mathrm{BV}^{+} \mathbf{G}_{2}^{1}(\underline{U})=\lim _{\Omega \ni \underline{Y} \rightarrow \underline{U}} \mathbf{G}_{2}^{1}(\underline{Y})=\mathcal{C}^{+}\left[\left.\mathbf{G}_{2}^{1}\right|_{\Gamma}\right](\underline{U})=(-1)^{n(n+1) / 2}(2 i)^{n}\left(\left.\frac{1}{2} \mathbf{G}_{2}^{1}\right|_{\Gamma}(\underline{U})+\frac{1}{2} \mathcal{S}\left[\left.\mathbf{G}_{2}^{1}\right|_{\Gamma}\right](\underline{U})\right) .
$$

In view of the assumption made, we thus have

$$
\mathrm{BV}^{+} \mathbf{G}_{2}^{1}=\frac{1}{2}(-1)^{n(n+1) / 2}(2 i)^{n}\left(\left.\mathbf{G}_{2}^{1}\right|_{\Gamma}+\left[\left.\mathbf{G}_{2}^{1}\right|_{\Gamma}\right] \mathcal{S}\right)=\left[\left.\mathbf{G}_{2}^{1}\right|_{\Gamma}\right] \mathcal{C}^{+}
$$

Now put $\mathbf{F}_{2}^{1}=\mathrm{BV}^{+}\left[\mathbf{G}_{2}^{1}\right] \mathcal{C}$. Then $\mathbf{F}_{2}^{1}$ is right $\mathbf{H}$-monogenic in $\Omega$ and belongs to $\mathcal{C}^{0, \alpha}(\Omega \cup \Gamma)$, with

$$
\mathrm{BV}^{+} \mathbf{F}_{2}^{1}(\underline{U})=\lim _{\Omega \ni \underline{Y} \rightarrow \underline{U}} \mathbf{F}_{2}^{1}(\underline{Y})=\left[\left.\mathbf{G}_{2}^{1}\right|_{\Gamma}\right] \mathcal{C}^{+} \mathcal{C}^{+}(\underline{U})=\left[\left.\mathbf{G}_{2}^{1}\right|_{\Gamma}\right] \mathcal{C}^{+}(\underline{U})=\mathrm{BV}^{+} \mathbf{G}_{2}^{1}(\underline{U}) .
$$

Next, put $\mathbf{H}_{2}^{1}=\mathbf{G}_{2}^{1}-\mathbf{F}_{2}^{1}$. Then $\mathbf{H}_{2}^{1}$ is harmonic in $\Omega$ and it belongs to $\mathcal{C}^{0, \alpha}(\Omega \cup \Gamma)$ with $B V^{+} \mathbf{H}_{2}^{1}=0$ on $\Gamma$. The Dirichlet problem for harmonic matrix functions then implies that $\mathbf{H}_{2}^{1} \equiv 0$ in $\Omega$ or $\mathbf{G}_{2}^{1}=\mathbf{F}_{2}^{1}$ in $\Omega$. Consequently, $\mathbf{G}_{2}^{1}$ is also right $\mathbf{H}$-monogenic in $\Omega$.

In the next theorem, we will show how the requirement $\boldsymbol{\Phi}_{\left(\underline{V}, \underline{V}^{\dagger}\right)}\left[\mathbf{G}_{2}^{1}\right](\underline{Y})=\mathbf{O}$ in the formulation of Theorem 6.1 can be avoided.

Theorem 6.2. Let $\mathbf{G}_{2}^{1}: \bar{\Omega} \rightarrow \mathbb{C}_{2 n}$ be a continuous matrix function with trace $\left.\mathbf{G}_{2}^{1}\right|_{\Gamma} \in \mathcal{C}^{0, \alpha}(\Gamma)$. Then $\mathbf{G}_{2}^{1}$ is two-sided $\mathbf{H}$-monogenic in $\Omega$ if and only if it is harmonic in $\Omega$ and

$$
\mathcal{S}\left[\left.\mathrm{G}_{2}^{1}\right|_{\Gamma}\right]=\left.\mathrm{G}_{2}^{1}\right|_{\Gamma}=\left[\left.\mathrm{G}_{2}^{1}\right|_{\Gamma}\right] \mathcal{S} .
$$


Proof. Suppose that $\mathrm{G}_{2}^{1}$ is two-sided $\mathrm{H}$-monogenic in $\Omega$. By Cauchy's integral formula (see [14]), we have that

$$
(-1)^{n(n+1) / 2}(2 i)^{n} \mathrm{G}_{2}^{1}(\underline{Y})=\mathcal{C}\left[\mathrm{G}_{2}^{1}\right](\underline{Y})=\left[\mathrm{G}_{2}^{1}\right] \mathcal{C}(\underline{Y}), \quad \underline{Y} \in \Omega
$$

The Plemelj-Sokhotzki formulae (see Theorem 4.3) then imply that

$$
\mathrm{G}_{2}^{1}(\underline{U})=\frac{1}{2}\left(\mathrm{G}_{2}^{1}(\underline{U})+\mathcal{S}\left[\mathrm{G}_{2}^{1}\right](\underline{U})\right)=\frac{1}{2}\left(\mathrm{G}_{2}^{1}(\underline{U})+\left[\mathrm{G}_{2}^{1}\right] \mathcal{S}(\underline{U})\right), \quad \underline{U} \in \Gamma .
$$

Consequently, $\mathcal{S}\left[\left.\mathrm{G}_{2}^{1}\right|_{\Gamma}\right](\underline{\mathcal{U}})=\left.\mathrm{G}_{2}^{1}\right|_{\Gamma}(\underline{\mathcal{U}})=\left[\left.\mathrm{G}_{2}^{1}\right|_{\Gamma}\right] \mathcal{S}(\underline{\mathcal{U}})$ for all $\underline{U} \in \Gamma$. Conversely, assume that $\mathbf{G}_{2}^{1}$ is harmonic in $\Omega$ and $\mathcal{S}\left[\left.\mathbf{G}_{2}^{1}\right|_{\Gamma}\right]=\left.\mathbf{G}_{2}^{1}\right|_{\Gamma}=\left[\left.\mathbf{G}_{2}^{1}\right|_{\Gamma}\right] \mathcal{S}$. Let us define the matrix functions

$$
\begin{array}{rll}
\mathbf{F}_{2}^{1}(\underline{Y})=\frac{1}{(-1)^{n(n+1) / 2}(2 i)^{n}} \mathcal{C}\left[\left.\mathrm{G}_{2}^{1}\right|_{\Gamma}\right](\underline{Y}), & \underline{Y} \in \Omega, & \mathbf{F}_{2}^{1}(\underline{U})=\left.\mathrm{G}_{2}^{1}\right|_{\Gamma}(\underline{U}), \quad \underline{\underline{U}} \in \Gamma, \\
\mathbf{H}_{2}^{1}(\underline{Y})=\frac{1}{(-1)^{n(n+1) / 2}(2 i)^{n}}\left[\left.\mathbf{G}_{2}^{1}\right|_{\Gamma}\right] \mathcal{C}(\underline{Y}), & \underline{Y} \in \Omega, & \mathbf{H}_{2}^{1}(\underline{U})=\left.\mathbf{G}_{2}^{1}\right|_{\Gamma}(\underline{U}), \quad \underline{U} \in \Gamma .
\end{array}
$$

They are left and right $\mathbf{H}$-monogenic, respectively, and hence both are harmonic in $\Omega$. Combining Theorems 4.3 and 4.4, we can assert that $\mathbf{F}_{2}^{1}$ and $\mathbf{H}_{2}^{1}$ are also continuous on $\bar{\Omega}$. As $\mathbf{F}_{2}^{1}-\mathbf{H}_{2}^{1}$ is harmonic in $\Omega$ and $\left.\mathbf{F}_{2}^{1}\right|_{\Gamma}=\left.\mathbf{H}_{2}^{1}\right|_{\Gamma}$, it follows that $\mathbf{F}_{2}^{1}(\underline{Y})=\mathbf{H}_{2}^{1}(\underline{Y})$ for $\underline{Y} \in \Omega$. The proof is completed by showing that $\mathbf{G}_{2}^{1}=\mathbf{F}_{2}^{1}=\mathbf{H}_{2}^{1}$ in $\Omega$.

\section{Main theorem}

Theorem 7.1. Let $\mathbf{G}_{2}^{1} \in \mathcal{C}^{0, \alpha}(\Gamma)(0<\alpha<1)$. Then the following statements are equivalent:

(i) $\mathrm{G}_{2}^{1}$ can be decomposed as in (5.1), the components $\mathrm{G}_{2}^{1^{ \pm}} \in C^{0, \alpha}(\Gamma)$ being extendable to two-sided $\mathbf{H}$-monogenic functions in $\Omega^{ \pm}$, vanishing at infinity;

(ii) $\mathcal{C}\left[\mathbf{G}_{2}^{1}\right]$ is a two-sided $\mathbf{H}$-monogenic in $\mathbb{R}^{2 n} \backslash \Gamma$.

Proof. Assuming (i) to hold, we may directly check, invoking Theorem 5.2, that $\mathcal{S}\left[\mathrm{G}_{2}^{1}\right]=$ $\left[\mathbf{G}_{2}^{1}\right] \mathcal{S}$. We thus get that $\mathcal{C}^{+}\left[\mathbf{G}_{2}^{1}\right]=\left[\mathbf{G}_{2}^{1}\right] \mathcal{C}^{+}$, which yields (ii) in view of Theorem 6.1 . This completes the proof since the inverse implication is trivial.

\section{Acknowledgments}

This paper was written while R. Abreu Blaya and J. Bory Reyes were visiting the Department of Mathematical Analysis of Ghent University. They were supported by the Special Research Fund no. 01T00807, obtained for the collaboration between the Clifford Research Group Ghent and the Cuban Research Group in Clifford analysis, on a project entitled Boundary Values Theory in Clifford Analysis. The authors wish to thank all members of this Department for their kind hospitality. 


\section{References}

[1] F. Brackx, R. Delanghe, and F. Sommen, Clifford Analysis, vol. 76 of Research Notes in Mathematics, Pitman, Boston, Mass, USA, 1982.

[2] R. Delanghe, F. Sommen, and V. Souček, Clifford Algebra and Spinor-Valued Functions, vol. 53 of Mathematics and Its Applications, Kluwer Academic Publishers, Dordrecht, The Netherlands, 1992.

[3] K. Gürlebeck and W. Sprößig, Quaternionic and Clifford Calculus for Physicists and Engineers, John Wiley \& Sons, Chichester, UK, 1998.

[4] J. E. Gilbert and M. A. M. Murray, Clifford Algebras and Dirac Operators in Harmonic Analysis, vol. 26 of Cambridge Studies in Advanced Mathematics, Cambridge University Press, Cambridge, UK, 1991.

[5] J. Ryan, "Complexified Clifford analysis," Complex Variables: Theory E Application, vol. 1, no. 1, pp. 119-149, 1982.

[6] R. Rocha-Chávez, M. Shapiro, and F. Sommen, Integral Theorems for Functions and Differential Forms in $\mathbb{C}^{m}$, vol. 428 of Chapman $\mathcal{E}$ Hall/CRC Research Notes in Mathematics, Chapman \& Hall/CRC, Boca Raton, Fla, USA, 2002.

[7] I. Sabadini and F. Sommen, "Hermitian Clifford analysis and resolutions," Mathematical Methods in the Applied Sciences, vol. 25, no. 16-18, pp. 1395-1413, 2002.

[8] F. Colombo, I. Sabadini, F. Sommen, and D. C. Struppa, Analysis of Dirac Systems and Computational Algebra, vol. 39 of Progress in Mathematical Physics, Birkhäuser, Boston, Mass, USA, 2004.

[9] F. Brackx, H. De Schepper, and F. Sommen, "The Hermitian Clifford analysis toolbox," accepted for Advances in Applied Clifford Algebras.

[10] F. Brackx, J. Bureš, H. De Schepper, D. Eelbode, F. Sommen, and V. Souček, “Fundaments of Hermitean Clifford analysis. I. Complex structure," Complex Analysis and Operator Theory, vol. 1, no. 3, pp. 341$365,2007$.

[11] F. Brackx, J. Bureš, H. De Schepper, D. Eelbode, F. Sommen, and V. Souček, "Fundaments of Hermitean Clifford analysis. II. Splitting of $h$-monogenic equations," Complex Variables and Elliptic Equations, vol. 52, no. 10-11, pp. 1063-1079, 2007.

[12] D. Eelbode, "Zonal Hermitean monogenic functions," in Proceedings of the 15th International Conference on Finite or Infinite Dimensional Complex Analysis and Applications (ICFIDCAA '07), Osaka, Japan, JulyAugust 2007.

[13] F. Sommen and D. Peña-Peña, "A Martinelli-Bochner formula for the Hermitian Dirac equation," Mathematical Methods in the Applied Sciences, vol. 30, no. 9, pp. 1049-1055, 2007.

[14] F. Brackx, B. De Knock, H. De Schepper, D. Peña-Peña, and F. Sommen, “On Cauchy and MartinelliBochner integral formulae in Hermitean Clifford analysis," submitted.

[15] R. Abreu-Blaya, J. Bory-Reyes, and D. Peña-Peña, "Jump problem and removable singularities for monogenic functions," The Journal of Geometric Analysis, vol. 17, no. 1, pp. 1-13, 2007.

[16] F. Brackx, B. De Knock, and H. De Schepper, "A matrix Hilbert transform in Hermitean Clifford analysis," Journal of Mathematical Analysis and Applications, vol. 344, no. 2, pp. 1068-1078, 2008.

[17] G. David and S. Semmes, Analysis of and on Uniformly Rectifiable Sets, vol. 38 of Mathematical Surveys and Monographs, American Mathematical Society, Providence, RI, USA, 1993.

[18] H. Federer, Geometric Measure Theory, Die Grundlehren der Mathematischen Wissenschaften, Band 153, Springer, New York, NY, USA, 1969.

[19] R. Abreu Blaya, D. Peña-Peña, and J. Bory Reyes, “Clifford Cauchy type integrals on Ahlfors-David regular surfaces in $\mathbb{R}^{m+1}$," Advances in Applied Clifford Algebras, vol. 13, no. 2, pp. 133-156, 2003.

[20] R. Abreu-Blaya, J. Bory-Reyes, O. F. Gerus, and M. Shapiro, "The Clifford-Cauchy transform with a continuous density: N. Davydov's theorem," Mathematical Methods in the Applied Sciences, vol. 28, no. 7, pp. 811-825, 2005.

[21] R. Abreu Blaya, J. Bory Reyes, and T. Moreno García, “Minkowski dimension and Cauchy transform in Clifford analysis," Complex Analysis and Operator Theory, vol. 1, no. 3, pp. 301-315, 2007.

[22] R. Abreu-Blaya, J. Bory-Reyes, and T. Moreno García, "Cauchy transform on nonrectifiable surfaces in Clifford analysis," Journal of Mathematical Analysis and Applications, vol. 339, no. 1, pp. 31-44, 2008.

[23] J. Bory Reyes and R. Abreu Blaya, "Cauchy transform and rectifiability in Clifford analysis," Zeitschrift für Analysis und ihre Anwendungen, vol. 24, no. 1, pp. 167-178, 2005.

[24] R. Abreu Blaya, J. Bory Reyes, D. Peña-Peña, and F. Sommen, "A boundary value problem for Hermitian monogenic functions," Boundary Value Problems, vol. 2008, Article ID 385874, 7 pages, 2008.

[25] R. Abreu Blaya, J. Bory Reyes, R. Delanghe, and F. Sommen, "Cauchy integral decomposition of multivector valued functions on hypersurfaces," Computational Methods and Function Theory, vol. 5, no. 1, pp. 111-134, 2005. 